\title{
A rare case of collodion baby and harlequin ichthyosis
}

\author{
Majid Mansouri ${ }^{1}$, Maryam Seifmanesh ${ }^{2}$, Siros Hemmatpour ${ }^{3}$, Farrokh $\operatorname{Rad}^{4}$, \\ Abdollah Sedaghat ${ }^{5}$, Banafshe Sedaghat ${ }^{5}$ \\ ${ }^{I}$ Neonatologist, Associated professor Kurdistan University of Medical Science, Sanandaj, Iran \\ ${ }^{2}$ Resident of Pediatrics, Kurdistan University of Medical Science,Sanandaj, Iran(Corresponding author) \\ ${ }^{3}$ Neonatologist,Assistant professor Kurdistan University of Medical Science, Sanandaj, Iran \\ ${ }^{4}$ Dermatologist, Associated professor Kurdistan University of Medical Science,Sanandaj, Iran \\ ${ }^{5}$ Resident of Pediatrics, Kurdistan University of Medical Science,Sanandaj, Iran
}

\begin{abstract}
A one day old female neonate by gestational age of 35 weeks, has clinical features of both collodion baby and harlequin ichthyosis. Coincidence of these two disorders is very rare. Prenatal diagnosis with ultrasonography, fetal skin biopsy, examination of cell from amniotic fluid is possible and can lead to early diagnosis before birth. Morbidity and mortality can reduce by appropriate and immediate treatment.
\end{abstract}

Key word: harlequin ichthyosis, collodionbaby,ectropion, eclabium

\section{Introduction}

Harlequin ichthyosis and collodion baby are disorders of cornification.They are a primary group of inherited conditions characterized clinically by patterns of scaling and histopathologically by hyperkeratosis. This disorders may manifest in the first weeks of life.Harlequinicthyosis is a rare very severe disorder of keratinization,and inherited as an autosomal recessive trait (usually), autosomal dominant trait or may be sporadic. Collodion baby represent a phenotypic expression of several genotypes and is less severely affected than infant with harlequin icthyosis.The common types of autosomal recessive inherited icthyosis are lamellar icthyosis and congenital icthyosiformerythrodema(nonbullous congenital icthyosiformerythroderma) $(1,2)$.
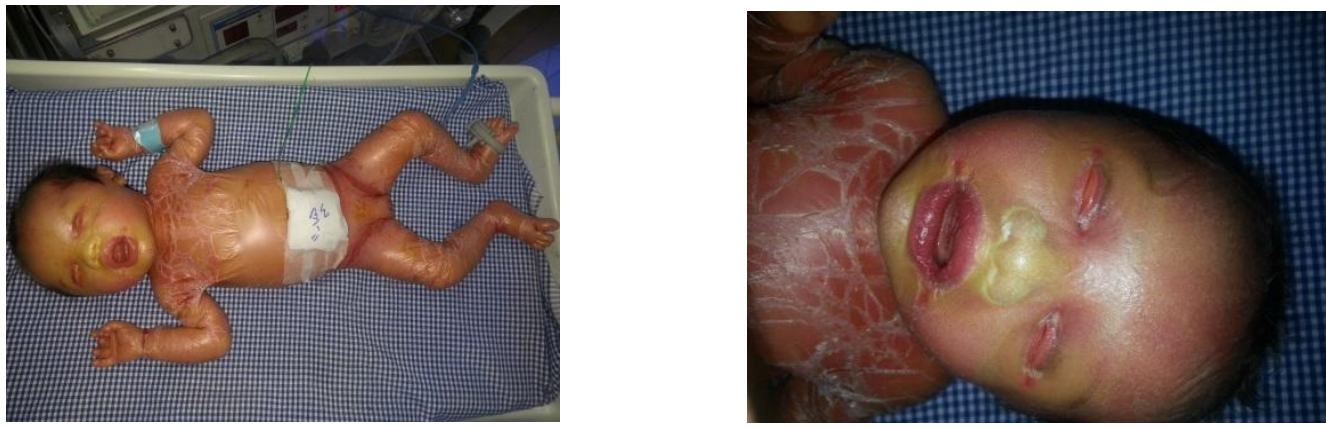

Fig 1 \& 2Parchement like skin with cracks, Ectropion and Eclabium( Fish Like Mouth)

\section{Case Report}

We present a one day female neonate, the first child of a non-consanguineously married couple, by gestational age of 35 weeks from 32 years old mother with history of infertility, three unsuccessful In-vitroFertilization (IVF) and a recent pregnancy due to IVF.No any abnormality was detected in the prenatal cares. On examination, the neonate had a weight of 2700 grams. Systemic examinations revealed ectropion in both eyes and fish like mouth (eclabium). Also lackof eyelashes and eyebrows as well as joint stiffness were noted.Skin was parchment like feel, shiny, brownish- yellow colour, with fissures in joints, intercostals and flexor areas. Nose and earswereflat. Hair was thin (Fig $1 \& 2$ ).Because of eclabiumand joint stiffness, the neonatal reflexes does not elicit.Theneonatehospitalizedin neonate intensive care unit(NICU) ward and monitored regarding temperature, hydration status and electrolytes balance. Prophylacticantibiotics, adequate hydration and nutrition were given to neonate. Bland lubricants were applied to the skin. Shedding of the collodionmemberane took place on the sixth day of birth without using keratolyticagents.Symptoms were improved gradually(Fig $3 \& 4$ ). The patient was discharged with a good condition. Seven-months follow up was normal. 

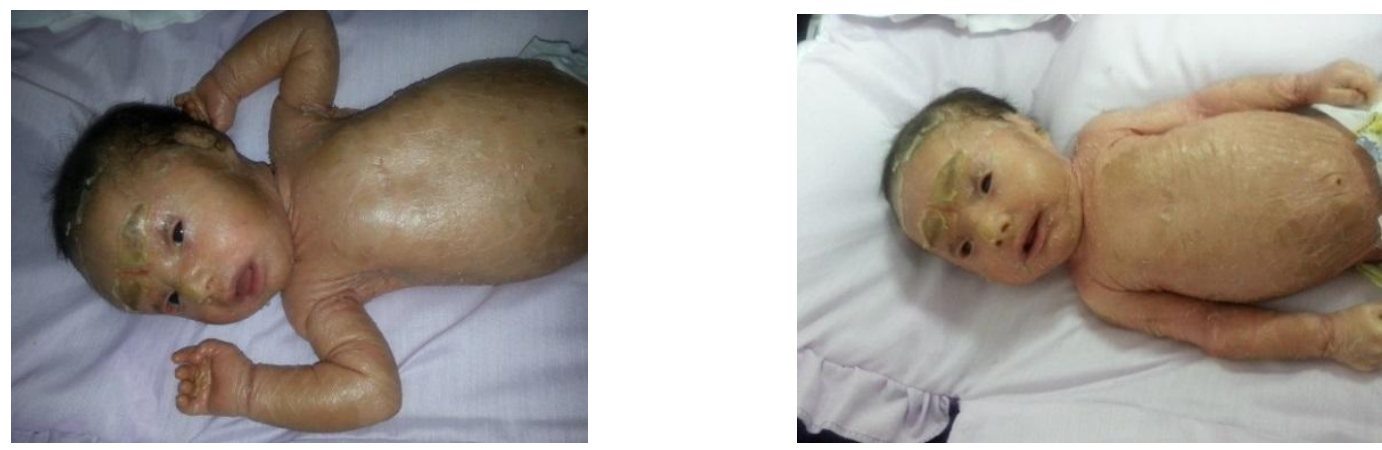

Fig 3 \& 4 Baby in 45 days of life

\section{Discussion}

The termichthyosis derives from the similarity of the skin condition to scales of a fish. Harlequin ichthyosisis a rare, very severe hereditary disorder of keratinization. Thisdisorderinherited as an autosomal recessive trait in most families, although a dominant form may exist and sporadic cases occur frequently. This disorder is caused by mutations in the ABCA12 gene, leads to defective lipid transport and ABCA12 activity that is essential for the development of the normal skin barrier. The skin of affected baby at birth, markedly thickened, ridged, and cracked forms horny plates over entire body, disfiguring the facial features and constricting the digits. Rigidity of the skin around eyes results in marked ectropion, although the globe is usually normal. The nose and ears are flattened, and the lips are everted and gaping, producing a "fish-mouth" deformity. The fissures are most prominent over areas of flextion. Most harlequin fetuses are born prematurely usually between 32 and 36 weeks of gestation, adding to their morbidity and mortality(1,2). Survival in the neonatal period is rare (3). Most die within the first days to weeks of life,but patients occasionally survive beyond infancy and have severe ichthyosis usually resembling lamellar ichthyosis or congenital ichthyosis form erythroderma (1).

Collodion baby is a common phenotype to several diseases including to variety of icthyosis or as an isolated and self limiting condition (4). The first clinical description of collodion membrane by Perez in 1880 continues to be valid: "the baby's skin in replaced by a cornifiedsubstance of uniform texture, which gives the body a varnished appearance" $(5,6)$. Only about 270 cases of collodion babies have been reported in literature since 1892, when the term first introduced by Hallopeau and Watelet (7).The babies are usually covered by a thick,taut membrane resembling oiled parchment or collodion at birth, which is subsequently shed. Rarely shedding of the collodion membrane results in a normal underlying integument. Prematurity is usual in collodion babies. Affected neonates have ectropion, flattening of the ears and nose, and fixation of the lips in an O-shaped configuration.Hair may be absent or may perforate the abnormal covering. The membrane cracks with initial respiratory efforts and, shortly after birth, begins to desquamate in large sheets. Complete shedding may take several weeks, and a new membrane may occasionally form in localized areas.Pedigree information and histopathologic examination of a skin biopsy are additional aids in the delineation of the specific type of ichthyosis (1, 4, 8-11). Approximately, 1:225 individual are a carrier and the risk to future pregnancies is $25 \%$. Risk of being a disease gene carrier in unaffected sibling of an affected individual is $2 / 3$ (12). The case that reported has clinical manifestations of both harlequin ichthyosis and collodionbaby.complications include sepsis, distal gangrene, and difficulties with feeding and respiration. Neonatal morbidity and mortality may be due to cutaneous infection (gram- positive and candida spp), aspiration pneumonia (squamous material), hypothermia, or hypernatremic dehydration. The outcome is uncertain, and accurate prognosis is impossible with respect to the subsequent development of ichthyosis $(1,2,13-15)$.Initial treatment includes high fluid intake to avoid dehydration from transepidermal water loss. Use of a humidified heated incubator, emulsifying ointments, careful attention to hygiene and oral retinoids $(1 \mathrm{mg} / \mathrm{kg} / \mathrm{day})(1-2)$. Shedding of membrane may facilitate by application of non occlusive lubricants and a high- humidity environment. Application of lubricant befor drying and using bath oils can prolonged hydration and softening.To enhance corneocytedisadhesion, keratolyicagents( salicylic acid, alpha hydroxyl acid), creams and lotions containing urea, and glycolic acid are used (16).

Management of ichthyosis patients in the newborn period runs the gamut from amelioration of mild cosmetic problems with excessive dryness and scaling to treatment of potentially life- threatening illness due to deficits in the epidermal barrier and subsequent infection. Signs and symptoms such as dryness, scalling, fissures and erosions, skin inflammation, keratoderma, erythema, secondary infections, pruritus, Anhidrosis, stiffness of the skin and ectropion are targets for ichthyosis therapy(2).In Harlequinichthyosis, prenatal diagnosis has been accomplished by fetoscopy, fetal skin biopsy, microscopic examination of cell from amniotic 
fluid, and ultrasonography(17). As part of multidisciplinary treatment, children need psychological support as they grow so that they accept their disease. It is also needed to reeducated those persons closest to the patient so that these children can pursue normal activities $(18,1)$.Parents are referred to an extensive online support group for families of infants with cornification disorders, the foundation for ichthyosis and related skin type (FIRST)( 2).

\section{Conclusion}

Coincidence of these two disorders is very rare. Parental ultrasonography, fetal skin biopsy, microscopic examination of cell from amniotic fluid can lead to early diagnosis before birth of these disorders. Morbidity and mortality can reduce by appropriate and immediate treatment.

\section{References}

[1] JG. Morelli, Disorders of keratinization, In: Behrman RE, Kliegman RM, Jenson HB (eds) Nelson Textbook Of Pediatrics, 19th edn. Philadelphia, WB Saunders. 2011, 2267-73.

[2] SB. HoathandV. Narenderan, The skin. In: Richard J. Martin, Avroy A. Fanaroff, and Michele C. Walsh. Neonatal-Perinatal Medicine, 9thEdition. 2010, 1713-16.

[3] S. Burdette-Taylor, Case study: harlequin fetus, a disease of cornification,Ostomy Wound Manage,1994;40:14 - 19.

[4] RK. Gupta SD. Sharma andR. Gupta,ColloidionBaby,Journal of Medical Education and Research,2009, 11: 204 - 205.

[5] D. Van GyselRL. LijnenSS. MoektiPC. De LaatandAP. Oranje,"Collodion baby: a follow-up study of 17 cases, Journal European Academy of Dermatology and Venereology, 2002, 16: 472-475.

[6] DY. ShrikhandeR. Chatterjee A. Kumar I. KocharK. Mandade and M. Vasoy, Collodion baby: A rare case report, Journal of Dental and Medical Science,2014, 13: 85-87.

[7] MR.Judge, "Collodion baby and Harlequin ichthyosis," in Textbook of Pediatric ermatology, Harper J, OranjeA, Prose N, Eds., pp. 118-125, Blackwell, Malden, Mass, USA, 2nd edition, 2006.

[8] EB. Lane Mclean WH. Keratins and skin disorders,Journal Pathology,2004, 204: 355-66.

[9] S. RudrappaB. ShambulingappaH V. Kumar,Colloidion baby, Asian Journal of Pediatric Practice,2005, 8: 54-56.

[10] DV. GyselRL. LinjenSS. Moeki,Colloidion baby: a follow up of 17 cases,Journal European AcadDermatolVenereol,2002, 16:47275.

[11] P. Pongprasit,Collodion baby: the outcome of long term follow up. Journal Medical Associated Thai.1993; 76:17-22.

[12] M. HartingN. Brunetti-PierriandCS. Chan et al,Selfhealingcollodion membrane and mild nonbullous congenital ichthyosis form erythroderma due to 2 novel mutations in the ALOX12B gene,Archives of Dermatological Research, 2008,144: 351-56.

[13] GR. Rodríguez PR. BelmanAH. Dander and MW. Cruz,Ictiosisautosómicarecesiva: informe de 4 casosdelsur de Veracruz,Bol Med Hosp Infant Mex, 2002,59:372-78.

[14] D.VanGyselRLP. LijnenSS. MoektiPCJ. De LaatandAP. Oranjet,Collodion baby: a follow-up study of 17 cases,JournalEurAcadDermVenereol,2003, 16:472-75.

[15] M. AkcakusT. GunesS. KurtogluandA. Ozturk,Collodion baby associated with asymmetric crying facies: a case report,Pediatrics Dermatology,2003,20:134-36.

[16] E. Frenk, A spontaneously healing colloidion baby: A light and electron microscopical study,ActaDermVenerol,1981, 61: 168-71.

[17] R. Sarkar AK. PatwariandAK. Dutta, An appraisal of pediatric dermatologic emergencies,IndianPediatrics,2000,37: 425-29.

[18] JP. O'Connell, A collodionbaby,Proceedings of the Royal Society of Medicine, 1977, 70: 212-213.

[19] AG. Estrada-LópezGA. García-MirandaandDM. Meza-Zamora,Collodionbaby,Boletin medico del Hospital Infantil de Mexico, 2010, 67 (4): 352- 358 